\title{
Lycopene improves the distorted ratio between AA/DHA in the seminal plasma of infertile males and increases the likelihood of successful pregnancy
}

\author{
Radka Filipcikova ${ }^{\text {a\# }}$, Ivana Oborna ${ }^{\text {b\# }}$, Jana Brezinovaa , Jiri Novotnyc, Gabriella Wojewodkad, Juan Bautista De Sanctise, \\ Lenka Radova ${ }^{f}$, Marian Hajduch', Danuta Radzioch ${ }^{\mathrm{d}}$
}

\begin{abstract}
Aims. Male infertility has been associated with imbalance in the polyunsaturated fatty acids arachidonic acid/docosahexaenoic acid (AA/DHA) ratio. This study aimed to assess whether use of the potent antioxidant, lycopene, would affect this imbalance in seminal plasma and to test whether modulation of the AA/DHA ratio correlates with male fertility.

Methods. The study included 44 males from infertile couples who were treated with lycopene for three months before scheduled IVF treatment. The control group was 13 fertile males who were also treated with lycopene for three months. $A A$ and DHA levels were measured in seminal plasma before and after treatment. The outcome of IVF treatment after lycopene use in of males from infertile couples was recorded.

Results. The subjects were subdivided into 21 normozoospermic males from couples with idiopathic infertility and 23 males with semen abnormalities. Prior to the treatment with lycopene, the AA/DHA ratios in both subgroups of patients were significantly higher than in fertile controls and improved following treatment with lycopene. Improvement was more marked in the normospermic males. Seven spontaneous pregnancies (16\%) occurred before the scheduled IVF treatment and 15 couples (42\%) achieved pregnancy after IVF.
\end{abstract}

Conclusions. Three months of treatment with lycopene led to a significant improvement in the AA/DHA ratio in seminal plasma of males from infertile couples and facilitated the spontaneous as well as IVF conception.

Key words: lycopene, oxidative stress, male infertility, seminal plasma, DHA, AA

Received: July 22, 2012; Accepted with revision: January 17, 2013; Available online: February 25, 2013

http://dx.doi.org/10.5507/bp.2013.007

${ }^{a}$ Department of Anatomy, Faculty of Medicine and Dentistry, Palacky University Olomouc, Czech Republic

${ }^{b}$ Department of Obstetrics and Gynecology, Faculty of Medicine and Dentistry, Palacky University Olomouc and University Hospital

Olomouc

'Department of Biology, Faculty of Medicine and Dentistry, Palacky University Olomouc

${ }^{d}$ Departments of Human Genetics, McGill University, Montreal, Canada

eInstitute of Immunology, Faculty of Medicine, Universidad Central de Venezuela, Caracas, Venezuela

fInstitute of Molecular and Translational Medicine, Faculty of Medicine and Dentistry, Palacky University Olomouc

Corresponding author: Ivana Oborna, e-mail: ivana.oborna@upol.cz

"The authors contributed equally to the work

\section{BACKGROUND}

Oxidative stress (OS), an imbalance between prooxidants and antioxidants, is considered an important contributing factor in many pathological conditions, including male infertility. Spermatozoa are susceptible to oxidative damage caused by reactive oxygen species (ROS). It has been demonstrated that excessive levels of ROS lead to lipid peroxidation of polyunsaturated fatty acids (PUFAs) which maintain the fluidity and integrity of plasma membranes ${ }^{1}$.

Omega-3 fatty acid, docosahexaenoic acid (DHA) is particularly susceptible to peroxidation due to its six double bonds. DHA is stored in phospholipids and triglycerides and has been shown to have anti-inflammatory properties $^{2}$. In contrast to DHA, excessive amounts of the omega-6 fatty acid, arachidonic acid (AA) have been shown to have pro-inflammatory effect. Although AA has also been found to play important roles in sperm capacitation and the acrosome reaction ${ }^{3}$, tight regulation of $\mathrm{AA}$ levels is essential because the balance between AA and
DHA may affect the functional capacity of spermatozoa. An imbalance in AA/DHA can also be caused by systemic inflammation which can be observed in many diseases ${ }^{4}$. Safarinejad et al. found significantly higher AA/DHA ratios in blood serum and spermatozoa of infertile men than fertile normozoospermic males 5 .

Spermatozoa are protected against excessive levels of ROS by both enzymatic and non-enzymatic antioxidants in seminal plasma. However, the imbalance leading to OS may result in damage to the sperm's DNA (ref. ${ }^{6}$ ), which has significant genetic and developmental consequences. The association of semen abnormalities such as oligozoo- and asthenozoospermia with OS has been extensively documented ${ }^{7,8}$. However, OS may also occur in normozoospermic males from infertile couples and this may account for previously unexplained infertility that might otherwise be attributed to the female partner ${ }^{9}$. For this reason antioxidants including vitamin $\mathrm{C}$, followed by vitamin $\mathrm{E}$, carnitine and many others, are frequently prescribed to improve male infertility, as reviewed in Lombardo et al. ${ }^{10}$. 
The carotenoid lycopene $\left(\mathrm{C}_{40} \mathrm{H}_{56}\right)$, found mainly in tomatoes ${ }^{11}$, is one of the most potent antioxidants. Under natural conditions, it exists primarily as a transisomer, but under the influence of heat, UV light, acid or oxygen it forms several cis-isomers, and both cis- and trans-forms can be detected in plasma and tissues. Apart from profound antioxidative action it has antiproliferative, immunomodulatory and antiinflammatory effects and promotes cell differentiation ${ }^{12}$. For these reasons, the role of lycopene in the prevention and treatment of various chronic diseases that may be caused by OS has been extensively studied ${ }^{13}$. Lycopene is bound to lipoproteins in blood plasma. The highest concentrations of lycopene were found in liver, testes, adrenal, prostate, adipose and breast tissues ${ }^{14}$. The results of the first study to examine the effects of lycopene on male infertility were published in 2002. Improvements in the semen parameters of men with unexplained infertility were found in about half of the subjects after three months of lycopene supplementation ${ }^{15}$. Daily dietary supplementation with tomato soup for two weeks significantly increased blood and seminal plasma levels of lycopene, but had no effect on the total radical scavenging capacity of semen ${ }^{16}$. Intake of the dietary supplement Menevit, containing vitamins $\mathrm{C}$ and E, selenium, zinc, folate, garlic and lycopene, for three months in a placebo-controlled study, led to a significant increase in clinical pregnancy rates after IVF/ICSI treatment $^{17}$.

The present study investigated the effects of lycopene supplementation for three months in (a) normozoospermic males from couples with idiopatic infertility, (b) males from the couples with solely male factor infertility and, (c) fertile volunteers who had spontaneously conceived children in the last two years. The main goal was to compare the changes in levels of two important phospholipid-bound polyunsaturated fatty acids, AA and DHA, and their ratios in seminal plasma from study and control subjects. The clinical objective of the study was to investigate the effect of such lycopene supplementation on the outcome of infertility treatment in the infertile couples included in our study.

\section{METHODS}

\section{Study design}

The study was approved by the Ethic Committee of University Hospital Olomouc and Faculty of Medicine and Dentistry of Palacky University Olomouc. All subjects gave their written consent. The study group consisted of 44 male partners in an infertile relationship (MPIR) referred to the program of assisted reproduction at the Department of Obstetrics and Gynecology, Faculty of Medicine and Dentistry, Palacky University Olomouc. All female partners of MPIR males were healthy. There were 21 couples with unexplained infertility (MPIR-NO) and 23 couples with known male factor infertility (MPIR-SA) such as mild to severe oligozoospermia, mild to severe asthenozoospermia and combinations of these conditions. The control group included 13 fertile male volunteers
(FV) who had fathered a child in a spontaneous conception within the previous 24 months.

Capsules containing $10 \mathrm{mg}$ of highly purified lycopene extracted from tomato puree (Brainway Inc, Prague, CR) were taken twice daily for three months. The lycopene capsules also contained $5.98 \mu \mathrm{mol}$ of vitamin $\mathrm{E}$ as a stabilizer. In all subjects, the first semen samples were taken before the first capsule of lycopene. A second set of semen samples was taken after three months of lycopene use.

\section{Laboratory analysis}

Semen samples were collected by masturbation following 3 to 5 days of sexual abstinence and were evaluated according to WHO criteria ${ }^{18}$. Seminal plasma was obtained by centrifugation of semen samples at $300 \mathrm{~g}$ for $7 \mathrm{~min}$ at room temperature. Seminal plasma samples were then stored in liquid nitrogen until analyzed. Lipids were separated according to the standard Folch method ${ }^{19}$. Briefly, $100 \mu \mathrm{L}$ of seminal plasma was stored in a $2: 1$ chloroform/ methanol mixture (v/v) with added $1 \mathrm{nM}$ butylated hydroxyanisole (BHA) to prevent oxidation. The samples were mixed with $1.9 \mathrm{~mL}$ of chloroform/methanol $(2: 1 \mathrm{v} / \mathrm{v})$ and $1 \mathrm{~mL}$ of cold water. The mixture was vortexed for 90 min at $4{ }^{\circ} \mathrm{C}$. From the resulting organic phase, aliquots were then used for phospholipid analysis by thin-layer chromatography (TLC), acid hydrolysis and methylation as described by Schlenk and Gellerman ${ }^{20}$. Lipids were separated from samples by TLC and detected by iodine. After scraping from the plate, the fatty acids were esterified. AA and DHA were identified using gas chromatography/mass spectrometry (GC/MS) (Hewlett Packard 5880 A with a WCOT capillary column, Supelco-10, $35 \mathrm{~m} \times$ $0.5 \mathrm{~mm}, 1 \mu \mathrm{m}$ film), and quantified using commercial standards (Sigma-Aldrich, Oakville, ON, Canada). The levels of AA and DHA in seminal plasma were expressed in $\mathrm{nmol} / \mu \mathrm{g}$ of protein. The aqueous phase of each sample was delipidized with diethylether and spun down. The protein pellet was then resuspended in $100 \mu \mathrm{L}$ of PBS. The protein content in each sample was then quantified by the bicinchoninic assay. Internal standards were used to control the recovery of proteins and lipids in each sample.

\section{Statistical analysis}

Values were expressed as median and $25^{\text {th }}$ and $75^{\text {th }}$ percentiles because of skewed distribution of variables. The Mann-Whitney test was used to test the differences between groups. Statistical significance was set at $P<0.05$ for all tests. All statistical analyses were performed using Statistica 8 (StatSoft, Inc.) software.

\section{RESULTS}

\section{Age and semen parameters}

The average age of the MPIR group was 33.6 years and of the FV group 34.1 years. In the MPIR group there were 21 normozoospermic males (MPIR-NO) and 23 males with semen abnormalities (MPIR-SA). All 13 volunteers from the FV group were normozoospermic. The semen 
parameters (semen volume, sperm count, sperm motility $(\mathrm{A}+\mathrm{B})$, sperm morphology, presence of leukocytes) were evaluated before and after lycopene administration. There were no significant changes in any of these parameters in either group.

Although 44 MPIR subjects entered the study, one normozoospermic male developed an allergic skin reaction during lycopene administration and withdrew from the study although there was no clear causal link between this reaction and lycopene.

\section{PUFA levels in seminal plasma before lycopene administration}

Prior to the treatment with lycopene, seminal plasma AA levels in the MPIR-SA subgroup were dramatically higher than those of the FV group $(P=0.00001)$. AA levels in the seminal plasma of patients from the MPIR-NO subgroup were higher than the seminal plasma of FV group of men, but the differences did not reach statistical significance $(P=0.055)$. We also found significantly higher levels of AA in the MPIR-SA subgroup than the MPIRNO subgroup $(P=0.018)$.

DHA levels in the seminal plasma of MPIR-SA subgroup of patients were lower than those in the seminal plasma of the FV group $(P=0.0002)$. There was also a trend towards lower levels of DHA in the seminal plasma of patients from MPIR-NO subgroup compared to the seminal plasma of FV group, but like AA, the difference did not reach statistical significance $(P=0.055)$. DHA levels in the seminal plasma of the MPIR-SA subgroup of patients were nonsignificantly lower than the levels in the plasma of the MPIR-NO $(P=0.09)$.

To evaluate the relative levels of the fatty acids for each patient, the AA/DHA ratio was calculated. Prior to lycopene treatment, both MPIR-NO and MPIR-SA subgroups displayed significantly higher AA/DHA ratios than FV patients $(P=0.02$ and $P=0.00002$, respectively $)$. The ratios of AA/DHA of the MPIR-SA subgroup were higher than those of the MPIR-NO subgroup $(P=0.07)$. The differences in the AA/DHA ratios between FV and MPIRSA groups were greater than between FV and MPIR-NO groups (Table 1).

\section{The effects of lycopene treatment on PUFA levels in seminal plasma}

Our study revealed a significant increase in DHA levels and even more significant decrease in AA levels, and thus a decrease in the AA/DHA ratio, in seminal plasma after three months of lycopene treatment in both MPIR groups. No significant changes in DHA, AA or AA/DHA ratio were found in the seminal plasma of the control FV group (Table 1 and 2, bottom lines). A remarkable decrease in the AA/DHA ratios in both MPIR subgroups was observed (Table 1 and 2). After three months of lycopene administration, the levels of AA, DHA and AA/DHA ratios in the MPIR-NO subgroup were completely indistinguishable from those of control FV group. However, while the aberrant levels of fatty acids improved in the MPIR-SA group following lycopene treatment, they were not completely normalized. Thus despite lycopene supplementation, the levels of AA, DHA and their ratio remained significantly different in the MPIR-SA group compared to the FV group.

\section{Infertility treatment outcome}

We also evaluated the clinical effect of lycopene on conception success. IVF treatment was carefully planned at the end of lycopene treatment or soon after. Nevertheless, surprisingly 7 couples (16\%) conceived spontaneously during lycopene intervention, before IVF treatment was scheduled. Six of these couples were classified as unexplained infertility (MPIR-NO), one had mild oligoasthenozoospermia (MPIR-SA). Of the remaining 36 couples who underwent IVF treatment, pregnancy was achieved in 15 couples (42\%). In 14 couples with unexplained infertility, eight pregnancies were achieved ( $57 \%$ ) and in 22 couples with male factor, seven pregnancies (32\%) were achieved. Altogether, of all 43 infertile couples 22 couples (51\%) conceived successfully, either spontaneously or after IVF treatment

We also compared the levels of AA, DHA and AA/ DHA ratios in males from couples who conceived with males from couples with IVF failure. No significant differences were found between males in the pregnancy group compared and the non-pregnancy group. Both experienced the same decrease in levels of $\mathrm{AA}$, increase in levels of DHA and consequently decrease in the AA/DHA ratios (Table 3 ).

\section{DISCUSSION}

Lycopene is a highly effective lipophilic antioxidant which is transported to and stored in the liver. Secondary organs, such as the prostate and testes, receive their lycopene supply from this store. Lycopene was found to accumulate in the cellular and intracellular membranes of prostasomes, which are integral parts of the prostatic secretions and constitute a significant fraction of human semen. In addition to its role as an antioxidant, lycopene is known to influence several biological processes such as cell communication, signalling and cell cycle progres$\operatorname{sion}^{12}$.

We have previously reported a positive effect of lycopene administration on DHA and AA and their ratio in normozoospermic men from infertile couples with excessive ROS production in washed spermatozoa. We have also found that the basal levels of phospholipid-bound PUFAs differed significantly between healthy fertile volunteers and infertile normozoospermic males, though there were no differences in their semen parameters ${ }^{21}$. Here we report, to our knowledge, for the first time, the effects of lycopene administration on AA, DHA and their ratios in the seminal plasma on the fertility potential in infertile couples with male factor or idiopatic infertility.

Devaraj et al..$^{22}$ tested the effects of daily dietary supplementation with $6.5,15$ or $30 \mathrm{mg}$ of purified lycopene over the course of 8 weeks on the blood lipid profile in a 

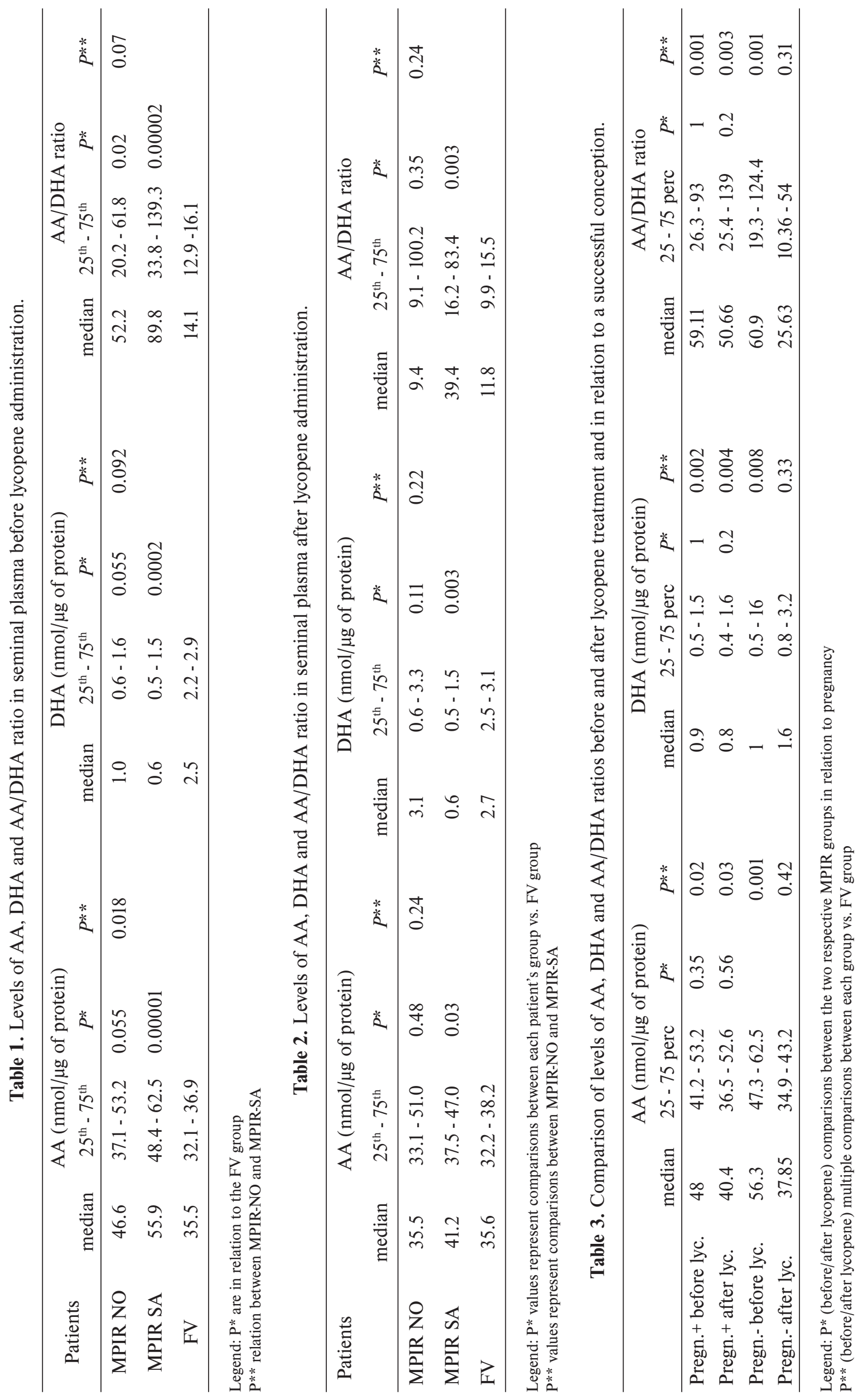
double-blind, randomized, placebo-controlled trial which included 77 healthy volunteers. The treatment increased the levels of lycopene in blood plasma compared to placebo. Despite these increases, no significant changes in lipid profiles or levels of other biomarkers of lipid peroxidation were observed. Only the subjects receiving 30 mg of lycopene per day exhibited significant decreases in DNA oxidative damage and urinary 8-hydroxydeoxyguanosine levels ${ }^{22}$.

Interestingly, carotenoid supplementation has recently been shown to have beneficial effects on semen quality in a bird model. The color of the breast plumage of male great tits is due to carotenoids. Consequently, males with more colorful plumage have increased protection against oxidative stress and have markedly better reproductive ability $^{23}$.

In addition to the effect of lycopene treatment on PUFA levels in seminal plasma, we have also found a positive effect on pregnancy rates in couples suffering from idiopatic or male factor infertility. The effects of lycopene were more pronounced in couples with idiopatic infertility where 6 spontaneous conceptions and 7 pregnancies after IVF were achieved during or soon after lycopene administration. Nevertheless, one spontaneous conception and 7 pregnancies after IVF in 22 couples with male factor infertility were also achieved.

Lenzi et al. ${ }^{24}$ studied the effects of long-term carnitine supplementation in the male partners from infertile couples. Although they observed no significant changes in the men's semen parameters, four pregnancies were achieved in the treatment group of 30 participants. Tremellen et al. ${ }^{17}$ observed a significant increase in clinical pregnancy rates after IVF/ICSI treatment following supplementation with Menevit for three months in a placebo controlled study. Our results support these findings that antioxidants, such as lycopene, have positive effects on natural conception and can increase clinical pregnancy rate in IVF treatment.

The effect of oxidative stress on fertility may depend on individual genetic backgrounds. It has been reported that gene polymorphisms can influence responses to antioxidant treatments. For example, polymorphisms $1704 \mathrm{G} / \mathrm{T}$ and $2184 \mathrm{~A} / \mathrm{G}$ in the RAGE gene are associated with differences in total carotenoid levels in the blood plasma of diabetic patients ${ }^{25}$. More studies are needed to elucidate the importance of these polymorphisms in the effects of lycopene for male infertility.

\section{CONCLUSIONS}

Our study reports for the first time the efficacy of lycopene in changing PUFA levels in seminal plasma and the ability of lycopene to restore fertility in a proportion of previously infertile couples. Lycopene was well-.tolerated and showed no proven side-effects in our study. This treatment may offer a solution to male infertility but more studies are needed to explain why some males regain fertility while others do not.

\section{ABBREVIATIONS}

AA, Arachidonic acid; BHA, Butylated hydroxyanisole; DHA, Docosahexaenoic acid; FV, Fertile volunteers; GC/MS, Gas chromatography/mass spectrometry; IVF/ ICSI, In vitro fertilization/ intracytoplasmic insemination; MPIR, Male partners from infertile relationship; MPIR-NO, Normozoospermic males; MPIR-SA, Males with semen abnormalities; OS, Oxidative stress; PBS, Phosphate buffered saline; PUFA, Polyunsaturated fatty acids; RAGE, Receptor of advanced glycation endproducts; ROS, Reactive oxygen species; TLC, Thin-layer chromatography.

\section{ACKNOWLEDGEMENT}

The study was supported by the grants of the International Grant Agency of the Ministry of Health of the Czech Republic No. A1 8621-5 and No. NT 11083.

The authors are grateful to M. Svobodova for documentation and to Dr. H. Fingerova for writing assistance. The lycopene extract was purchased from Brainway Inc., which did not provide any funding and was not involved in the design of the study, data collection, analysis or preparation of the manuscript.

The study was conceived by the authors. The conception and design of the study was performed by IO and $\mathrm{RF}$, the data were collected by IO, RF and JB, semen analysis was performed by JB, RF and JN, PUFA samples processing GW and DR and PUFA samples analysis by JBS, statistical evaluation of the data was done by LR, final approval was done by IO, MH and DR.

\section{REFERENCES}

1. Hossain S, Tareq KMA, Hammano K-I, Tsujii H. Effect of fatty acids on boar sperm motility, viability and acrosome reaction. Reprod Med Biol 2007;6:235-9.

2. Lukiw WJ, Bazan NG. Docosahexaenoic Acid and the Aging Brain. J Nutr 2008;138:2510-4.

3. Maccarrone M, Barboni B, Paradisi A, Bernabo N, Gasperi V, Pistilli MG, Fezza F, Lucidi P, Mattioli M. Characterization of the endocannabinoid system in boar spermatozoa and implications for sperm capacitation and acrosome reaction. J Cell Sci 2005; 118:4393-404.

4. Guilbault C, Wojewodka G, Saeed Z, Hajduch M, Matouk E, De Sanctis $J B$, Radzioch D. Cystic fibrosis fatty acid imbalance is linked to ceramide deficiency and corrected by fenretinide. Am J Resp Cell and Mol Biol 2009;41:100-6.

5. Safarinejad MR, Hosseini SY, Dadkhah F, Asgari MA. Relationship of omega- 3 and omega- 6 fatty acids with semen characteristics, and anti-oxidant status of seminal plasma: A comparison between fertile and infertile men. Clin Nutr 2010;29:100-5.

6. Agarwal A, Gupta S, Sharma R. Oxidative stress and its implications in male infertility - a clinician's perspective. Reprod Biomed Online 2005; 11:641-50.

7. Aitken RJ, Clarkson JS, Fishel S. Generation of reactive oxygen species, lipid peroxidation, and human sperm function. Biol Reprod 1989;41:183-97.

8. Sharma RK, Agarwal A. Role of reactive oxygen species in male infertility. Urology 1996; 48:835-50.

9. Pasqualoto FF, Sharma R, Kobayashi H, Nelson DR, Thomas AJ, Agarwal A. Oxidative stress in normospermic men undergoing infertility evaluation. J Androl 2001;22(2):316-22. 
10. Lombardo F, Sansone A, Romanelli F, Paoli D, Gandini L, Lenzi A. The role of antioxidant therapy in the treatment of male infertility: an overview. Asian J Androl 2011;13:690-7.

11. Hadley P, Schwartz SJ. Lycopene. In Encyclopaedia of dietary supplements. New York 2005:421-34.

12. Wertz K, Siler U, Goralczyk R. Lycopene: modes of action to promote prostate health. Arch Biochem Biophys 2004;430:127-34.

13. Lemineur T, Deby-Dupont G, Preiser JCH. Biomarkers of oxidative stress in critically ill patients: what should be measured, when and how? Curr Opin Clin Nutr Metab Care 2006;9(6):704-10.

14. Stahl W, Sies H. Perspectives in biochemistry and biophysics. Lycopene: A biological important carotenoid for humans. Arch of Biochem Biophys 1996;336:1-9.

15. Gupta NP, Kumar R. Lycopene therapy in idiopatic male infertility preliminary report. Int Urol Nephrol 2002;34(3):369-72.

16. Goyal A, Chopra M, Lwaleed BA, Birch B, Cooper AJ. The effects of dietary lycopene supplementation on human seminal plasma. BJU Int 2007;99(6):1456-60.

17. Tremellen K, Miari G, Froiland D, Thompson J. A randomised control trial examining the effect of an antioxidant (Menevit) on pregnancy outcome during IVF-ICSI treatment. Austr N Z J Obstet Gynaecol 2007;47(3):216-21.

18. WHO laboratory manual for the examination of human semen and sperm-cervical mucus interaction. Cambridge, University Press, Cambridge, UK, 1999.
19. Folch J, Lees M, Sloane SGH. Simple method for the isolation and purification of total lipids from animal tissues. J Biol Chem 1957;226(1):497-509.

20. Schlenk H, Gellerman J. Esterification of fatty acids with diazomethane on a small scale. Anal Chem 1960;32:1412-4.

21. Oborna I, Wojewodka G, De Sanctis JB, Fingerova H, Svobodova M Brezinova J, Hajduch M, Novotny J, Radova L, Radzioch D. Increased lipid peroxidation and abnormal fatty acid profiles in seminal and blood plasma of normozoospermic males from infertile couples. Hum Reprod 2010;25(2):308-16.

22. Devaraj S, Mathur S, Basu A, Aung HH, Vasu VT, Meyers S, Jialal I. A dose-response study on the effects of purified lycopene supplementation on biomarkers of oxidative stress. J Am Coll Nutr 2008;27(2):267-73.

23. Helfenstein F, Losdat S, Moller AP, Blount JD, Richner H. Sperm of colourful males are better protected against oxidative stress. Ecol Lett 2010;13:213-22.

24. Lenzi A, Sgro P, Salacone P, Paoli D, Gilio B, Lombardo F, Santulli M, Agarwal A, Gandini L. A placebo-controlled double blind trial of the use of combined L-carnitine and L-acetyl-carnitine treatment in men with asthenozoospermia. Fertil Steril 2004;81(6):1578-84

25. Kankova K, Marova I, Zahejsky J, Muzik J, Stejskalova A, Znojil V, Vacha J. Polymorphisms 1704G/T and 2184A/G in the RAGE gene are associated with antioxidant status. Metabolism 2001;50:1152-60. 